\title{
Electromagnetic wave beam self - guidance in a liquid crystal - semiconductor cell
}

\author{
A. Walczak ${ }^{*}$ \\ Military University of Technology, Kaliskiego 2, 00-908 Warszawa
}

Received June 07, 2010; accepted June 21, 2010; published June 30, 2010

\begin{abstract}
A theoretical model and numerical results for a new kind of optical waveguide has been presented. The discussed waveguide is optically self-induced during light beam propagation along the boundary between a liquid crystal and a semiconducting polymer in an electrically driven lc cell. Experimental results obtained earlier are briefly reported here, and the theoretical model is discussed. The exploited liquid crystal was of birefringence close to 0.4 . The photorefractive polymer was a poly (N-vinyl) carbazole doped with 2, 4, 7-trinitro-fluorenone.
\end{abstract}

Optical waveguide structures obtained in a thin liquid crystal (lc) film driven between two photo-chromic commands surfaces have been reported earlier by other authors [1]. This solution applies Langmuir-BlodgettKuhn monomolecular layers of a polymer with azobenzene side chains deposited on a glass sheet. When exposed to light of appropriate wavelength, the command surfaces undergo the trans-cis photo-isomerization process that induces a reversible change of orientation in the lc layer situated next to the command surface. Such a change of the lc layer orientation can create an optical waveguide along the way of an external beam illuminated monomolecular layer. The transition induced in lc layer is shown to be continuous, depending on the ratio of the cistrans chromophore concentration in the command surface. The kind of disadvantage there is due to the fact that the waveguide and light beam which induces the waveguiding path are not co-planar and cannot be arranged in an integrated device. The most innovative way seems to be a nonlinear waveguides array first introduced by Christodoulides et al. [2]. This concept has been widely developed by Assanto, and also by Assanto and Karpierz et al. [3-5]. The authors used the interaction of a laser beam with an lc layer driven by patterned electrodes forming light guiding channels. Unfortunately, it still seems be quite a long way from the present fiber net technology.

In reference to the mentioned results, we observed waveguide creation when an electrically driven lc layer is aligned on a photoconductive polymer (see Fig. 2). Light was guided at a relatively long distance $(\sim 15 \mathrm{~mm})$ with no observed beam widening due to refraction (see Fig. 1). It

*E-mail: awalczak@wat.edu.pl indicates that light creates a waveguide for itself, and no self focusing of light is observed. Because of good transparency of liquid crystal in all visible range up to near infrared spectrum the dominant losses during propagation are scattering losses. Because of good aligning of the $\mathrm{lc}$ in the strong electric field (range of $10^{6}$ $\mathrm{V} / \mathrm{m}$ ) we can assume that the number of optical heterogeneities of the lc layer caused scattering is relatively small. Experimental results have been presented and discussed earlier [7-9].

A theoretical model, as well as numerical results, is reported here. The presentation of the observed waveguide is shown in Fig. 1.

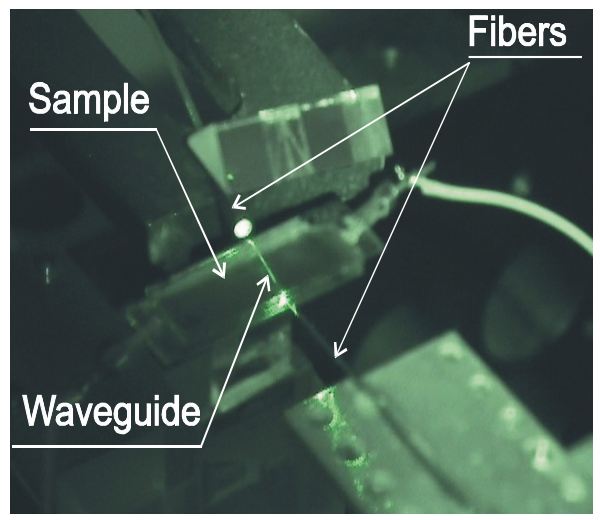

Fig. 1. Experimental observation of the self-induced waveguide [8].

The lc cell arrangement and the light path scheme are shown in Fig. 2. The layers collection is as follows: glass plate, indium-tin oxide (ITO), photoconductive polymer, lc, polyimide (PI), ITO, glass plate.

The sensitizer 2, 4, 7-trinitro-fluorenone (TNF) was applied as a dopant in the PVC photoconductive polymer layer. Both substances were produced by Aldrich. A liquid crystal nematic mixture with optical birefringence $\Delta \mathrm{n}$ equal to 0.38 was used in the sample. Refractive indices for this mixture were respectively: $\mathrm{n}_{\mathrm{o}}$ equal to 1.52, and $\mathrm{n}_{\mathrm{e}}$ equal to 1.9. The used mixture of $1 \mathrm{c}$ was prepared in the Institute of Chemistry MUT with a symbolic name W1662 [6]. Investigations were carried out on the set of lc cells filled with a W1662 mixture and 
prepared as $6 \mu \mathrm{m}$ thick. The thickness of an empty cell was controlled by means of an interferometer. The PVC layers were $100 \mathrm{~nm}$ thick. The initial orientation of the lc layer was planar (homogeneuos).

The lasers applied during experiments emit $p$ polarized $\mathrm{CW} 530$ and $532 \mathrm{~nm}$ wavelength radiation. The laser power was equal to $0.1 \mathrm{~mW}$. Doped PVC also changes its conductivity for illumination by the radiation of a wavelength equal to $632.8 \mathrm{~nm}$. Nevertheless, the sensitivity of exploited PVC is rather week for this wavelength.

Fibers ended with focusers (indexed 1 in Fig. 2 and visible in Fig. 1) were placed on a stage controlled by piezo-actuators with submicron accuracy of step, to be explored as tools for light beam insertion and dropping into and from the lc layer. The upper side of the sample was transparent and the light beam path was registered by means of a zoomed CCD camera. Observations were made in crossed polarizer. One polarizer was placed under the lc cell with the second polarizer placed on the camera. The laser beam path in the sample is shown schematically in Fig. 2, and its experimental observation in Fig. 1.

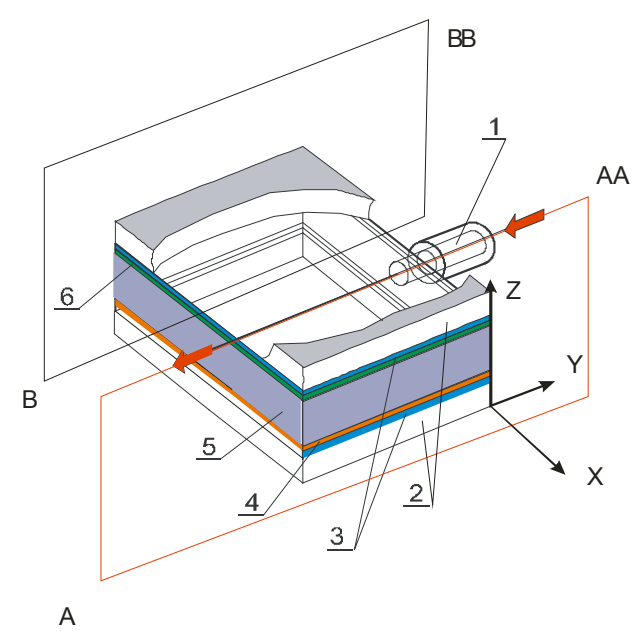

Fig. 2. Liquid crystal cell arrangement with asymmetric aligning layers. 1-fiber with focuser, 2- glass plates, 3- ITO electrodes, 4, 6aligning layers of polyimide (6) and PVC (4), 5- lc layer. Coordinates $\mathrm{x}, \mathrm{y}, \mathrm{z}$ as well as cross-sections A-AA, and B-BB added for results and discussion clarity.

The photo-induced charge generated by light beam of intensity $\mathrm{I}(\mathrm{x}, \mathrm{z})$ is distributed in the illuminated part of PVC layer and creates electric field called space charge field in accordance with formula (Kukhtarev model [10]):

$$
E^{s c}=\frac{k_{B} T}{e} \frac{1}{I(x, z)} \frac{\partial I(x, z)}{\partial x \partial z}
$$

Because the PVC layer is much thinner then the lc layer (over 50 times) we assumed that light intensity incident on the PVC layer was only x-dependent (see Fig. 2). So the formula for a space charge field is as follows:

$$
E^{s c}=\frac{k_{B} T}{e} \frac{1}{I(x)} \frac{d I(x)}{d x}
$$

The Kukhtarev model assumes one kind of carrier because of the very different mobility of holes and electrons in a doped photopolymer. The resulting shape of the electric field caused by a space charge in accordance with formula (2) is placed in Fig. 3 while steady illumination segment along $\mathrm{x}$ axis was assumed. Because of formula (2) a strong jump of $E^{s c}$ arises on both ends of the short illuminated segment of a thin PVC layer. It is shown in Fig. 3 as vertical arrows.

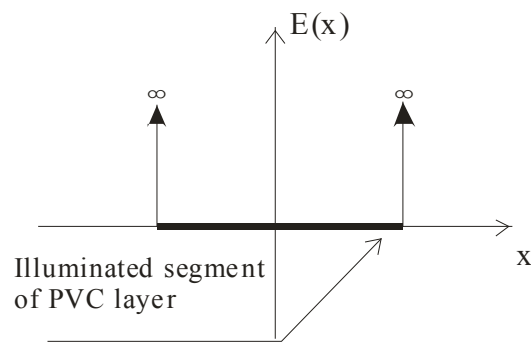

Fig. 3. Model of space charge field $E^{s c}$ induced in PVC layer with arrows underlined $\delta$-Dirac like behavior of $\mathrm{E}^{\mathrm{sc}}$ on the edges of illuminated segment.

The Ericksen-Leslie equation (ELE) for lc layer deformation is of the form:

$$
\begin{aligned}
& \left(k_{11} \cos ^{2} \theta+k_{33} \sin ^{2} \theta\right) \frac{\partial^{2} \theta}{\partial z^{2}}+\left(k_{33}-k_{11}\right) \\
& \sin \theta \cos \theta\left(\frac{\partial \theta}{\partial z}\right)^{2}+\varepsilon_{0} \Delta \varepsilon E^{2} \sin \theta \cos \theta=0
\end{aligned}
$$

The local electric field E value in formula (3) is caused in the illuminated area by the sum of external driving field $\mathrm{E}(\mathrm{z})$ and space charge electric field $\mathrm{E}(\mathrm{x})$. Numerical solution of ELE by the Finite Element Method (FEM) is presented in Fig. 4. The jump of $E^{s c}$ shown in Fig. 3 was assumed in calculations as a rectangular series limit of the Dirac distribution. The boundary conditions applied in calculations were assumed as strong anchoring of $1 \mathrm{c}$ molecules. Calculations were made for a 10-micrometer diameter of the light beam and 6-micrometer thickness of the lc cell. One can see that effective refraction index distribution inside an electrically driven lc layer along the beam path is of higher values than in the vicinity of this path. Let us take two cross sections A-AA and B-BB illustrated in Fig. 2 and Fig. 4. It means the cross-section along the beam path and beside it. The comparison of refractive index profiles in both cross - sections illustrates that there always exist conditions for 
waveguide creation in the area shown in Fig. 5. In all this area the refractive index in the illuminated beam path is higher than in its vicinity. Then the basic condition for waveguide existence is fulfilled. One can observe that the area shown in Fig. 5 and Fig. 4 near the cell walls always exists in the illuminated part of the lc cell. It means that at least two waveguides can be created in the described situation. It must be underlined that such a waveguide rises without any self focusing of light and nonlinear interaction between light and lc compounds. In summary, we created a theoretical model of phenomena which take place in an asymmetric lc cell with one aligning layer consisting of common polyimide, while the second consists of a photorefractive polymer. We observed the self - induction of a waveguide by a light beam propagated in such a cell along the lc-photopolymer boundary.

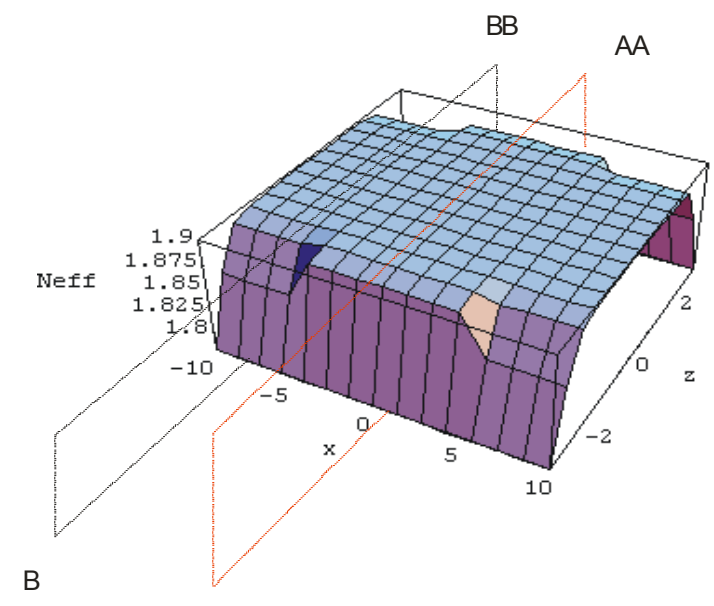

A

Fig. 4. The effective refractive index inside lc cell for light beam directed along y and constrained to beam aperture (illuminated PVC layer segment) and driving voltage equal to $1.2 \mathrm{~V}_{\text {th }}$ (threshold voltage denoted as $\mathrm{V}_{\text {th }}$ ).

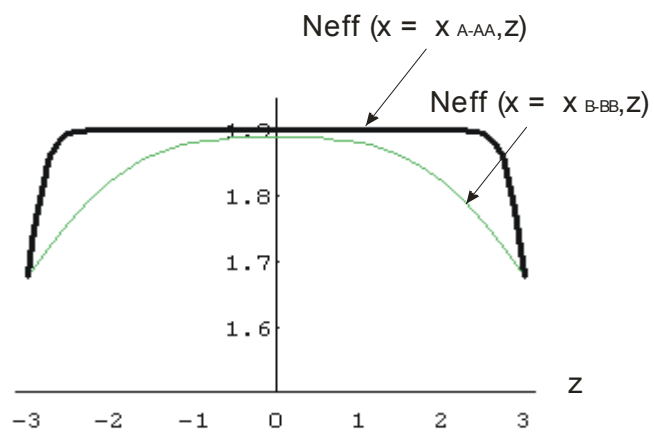

Fig. 5. Comparison of refractive index profiles in two cross-sections belonging to illuminated and not illuminated area appropriately A-AA and B-BB in Fig. 4.
Here we explained the influence of a space charge generated inside a photopolymer layer on a localized deformation induced in the lc layer and we proved that such a deformation causes conditions for light guidance.

As far as the author knows, it is the first model and explanation of light behavior in this kind of optical waveguide. The experimental results described earlier and the theoretical model presented here, together with numerical outcomes, composes data of a new kind of waveguide. At present we are continuing research for new semiconducting substrates as well as new liquid crystals to obtain controlled waveguide parameters. To achieve this aim, the Kukhtarev model should be also revised. Research work to get a polymer or another kind of substrate photosensitive in the near infrared spectrum is also continued.

\section{Acknowledgements}

Research is supported by the Department of State for Higher Education and Science in Poland, grant no. N N517228235.

\section{References}

[1] H. Knobloch, H. Orendi, M. Buchel, T. Seki, S. Ito, W. Knoll, J.Appl. Phys. 77, 481 (1995).

[2] D.N. Christodoulides, R.I. Joseph, Opt.Lett. 13, 794 (1988).

[3] A. Fratalocchi, G. Assanto, K.A. Brzdąkiewicz, M.A. Karpierz, Opt. Expr. 13, 1808 (2005).

[4] G. Assanto, A. Piccardi, A. Alberucci, S. Residori, U. Bortolozza, Phot. Lett. of Poland 1(4), 151 (2009).

[5] F. Sala, M. Karpierz, Phot. Lett. of Poland 1(4), 163 (2009).

[6] A. Spadlo, R. Dabrowski, J. Dziaduszek, S. Urban, E. Scibor, S. Gauza, S.T. Wu, J. Opt. Technol. 72, 659 (2005).

[7] A. Walczak, E. Nowinowski-Kruszelnicki, ECLC Conference, Lisbona 2007.

[8] A. Walczak, E. Nowinowski-Kruszelnicki, Liquid Crystal Photonic Workshop, Cambridge 2008.

[9] A. Walczak, E. Nowinowski-Kruszelnicki, Opt. Eng. 47(3) (2008).

[10] N.V. Kukhtarev, S.F. Lyuksyutov, P. Buchhave, T. Kukhtareva, K. Sayano, P.P. Benerjee, Phys. Rev. A 58, 4051 (1998). 